\title{
Pancreatoyeyunoanastomosis con técnica de Blumgart modificada para reconstrucción post-pancreatoduodenectomía. Estudio de serie de casos con seguimiento
}

\author{
Héctor Losada M. ${ }^{1,2,3}$, Sonia Curitol S. ${ }^{1}$, Andrés Troncoso ${ }^{1,2}$, \\ Hernán Herrera C. ${ }^{1,2}$, Jorge Silva A. ${ }^{2,3}$
}

\section{Pancreatojejunostomy with modified Blumgart technique for post-pancreatoduodenectomy reconstruction}

Introduction: Pancreatoduodenectomy is a complex surgery, with morbidity close to $30 \%$ and mortality between $1 \%$ and $5 \%$. The main contributing factor to morbidity and mortality is postoperative pancreatic fistula (POPF). At present, there is no globally standardized technique for pancreatic reconstruction. Aim: To determine the prevalence of clinically relevant POPF in a sample of patients who underwent pancreaticojejunal anastomosis reconstruction with Blumgart's modified technique for post-pancreatoduodenectomy reconstruction at Hospital Hernán Henríquez Aravena between 2014 and 2017. Material and Method: Case series with follow-up from july 2014 to april 2017. Patients who underwent pancreatic reconstruction with Blumgart's modified technique were included. The modification consisted of the use of Pledgets ${ }^{\circledR}$ (poly-tetrafluoro-ethylene) at the inicial points in páncreas with the idea of reducing the possibility of tissue tearing. We excluded patients who underwent another reconstruction technique. Clinically relevant POPF (grade B/C) was considered to asses morbidity. Descriptive statistics were used with measures of central tendency and dispersion. Results: Case series of 12 patients, $9(75 \%)$ were female and $3(25 \%)$ were male. The mean age was $59 \pm 8.5$ years. The morbidity was $25 \%$ and the rate of grade $\mathrm{B} / \mathrm{C}$ fistula was $0 \%$. All pancreatic fistulas were grade A, not clinically relevant. Conclusion: The Blumgart's modified technique seems to be a safe and reproducible technique for pancreticojejunal anastomosis.

Key words: pancreatic fistula; blumgart/pancreatoduodenectomy; pancreatic fistula score.

\section{Resumen}

Introducción: La pancreatoduodenectomía es una cirugía compleja, con cifras de morbilidad cercanas a $30 \%$ y mortalidad entre 1 a $5 \%$. El principal factor responsable de morbilidad y mortalidad es la fístula pancreática posoperatoria (FPPO). En la actualidad no existe una técnica universalmente estandarizada para la reconstrucción pancreática. Objetivo: Determinar la prevalencia de FPPO clínicamente relevante en una serie de pacientes en los que se realizó reconstrucción pancreática con pancreatoyeyunoanastomosis con técnica de Blumgart modificada para reconstrucción post-pancreatoduodenectomía en Hospital Hernán Henríquez Aravena entre los años 2014-2017. Material y Método: Serie de casos con seguimiento de julio de 2014 a abril de 2017. Se incluyeron pacientes a quienes se realizó reconstrucción pancreática con técnica de Blumgart modificada. La modificación consistió en el uso de pledgets $^{\circledR}$ (poli-tetrafluoroetileno) en los puntos iniciales en el páncreas con la idea de disminuir la posibilidad de desgarro del tejido. Se excluyeron pacientes a quienes se realizó otra técnica de reconstrucción. Se consideró FPPO clínicamente relevante (grado B/C) para evaluar morbilidad. Se utilizó estadística descriptiva con medidas de tendencia central y dispersión. Resultados: Serie de casos de 12 pacientes, 9 (75\%) de género femenino y $3(25 \%)$ de género masculino. La edad promedio fue de $59 \pm 8,5$ años. La morbilidad fue de $25 \%$ y la tasa de fístula grado $\mathrm{B} / \mathrm{C}$ fue $0 \%$. Todas las fístulas pancreáticas fueron grado $\mathrm{A}(33,3 \%)$, sin relevancia clínica. Conclusión: La técnica de Blumgart modificada parece ser una técnica segura y reproducible para pancreato-yeyuyoanastomosis.

Palabras clave: fístula pancreática; blumgart/pancreatoduodenectomía; escore fístula pancreática.
'Departamento de Cirugía. Universidad de La Frontera. Temuco, Chile.

Equipo de Cirugía

Biliopancreática. Hospital Regional de Temuco. Temuco, Chile.

${ }^{3}$ Equipo de Cirugía Biliopancreática. Departamento de Cirugía. Clínica Alemana de Temuco. Temuco, Chile.

Presentado en LXXXIX Congreso Chileno e Internacional de Cirugía, La Serena, 2016.

Presentado en Americas Hepato-Pancreato-Biliary Association (AHPBA) Annual Meeting, Miami, 2017.

Recibido el 18 de julio de 2017 y aceptado para publicación el 12 de septiembre de 2017.

Correspondencia a: Dr. Héctor Losada M. hector.losada@ufrontera.cl 


\section{Introducción}

La pancreatoduodenectomía (PD) es una de las técnicas más complejas de la cirugía hepatobiliopancreática. La mortalidad en los últimos años se ha acercado a cifras por debajo del $5 \%$. La morbilidad continúa alrededor del $35 \%$ y está asociada principalmente a la filtración de la anastomosis pancreática $^{1-10}$.

Nuestro equipo publicó la experiencia con reconstrucción pancreática en PD utilizando pancreato-gastro-anastomosis ducto mucosa (PGADM), con una morbilidad global del $35 \%$ y mortalidad de $5 \%{ }^{11}$. Sin embargo, algunos problemas conceptuales de la PGADM son la posibilidad de fístula de alto débito y la posibilidad de dehiscencia potencial asociada a la insuflación en el control endoscopio de pacientes con hemorragia digestiva alta cuando la causa son lesiones de estrés en la mucosa gástrica o duodenal, o hemorragia proveniente de la anastomosis ducto-mucosa.

La evidencia científica disponible no demuestra que una técnica sea superior a otra ${ }^{11-13}$.

Nuestro objetivo es determinar la prevalencia de fístula pancreática en una serie de pacientes a quienes se les realizó pancreatoyeyunoanastomosis con técnica de Blumgart modificada para reconstrucción post-pancreatoduodenectomía en Hospital Hernán Henríquez Aravena entre los años 2014- 2017.

\section{Materiales y Métodos}

\section{Diseño}

Estudio de serie de casos con seguimiento.

\section{Población}

Doce pacientes a quienes se realizó reconstrucción pancreática con técnica de Blumgart modificada, por el equipo de cirugía biliopancreática en el Hospital Hernán Henríquez Aravena (HHHA), entre julio de 2014 y abril de 2017.

\section{Criterios de exclusión}

Haber sido intervenidos con otra técnica de reconstrucción.

\section{Maniobra}

Todos los pacientes fueron evaluados en reunión multidisciplinaria del equipo de hígado y páncreas, donde se revisa historia clínica, imágenes de abdomen (usualmente resonancia magnética nuclear de páncreas y/o tomografía computada de abdomen) y los exámenes de laboratorio de los pacientes.
La técnica respectiva realizada por nuestro equipo ya se publicó en un reporte previo ${ }^{11}$.

La fase reconstructiva es realizada actualmente por el mismo equipo que realiza la fase resectiva y se utiliza siempre magnificación de 2,5X.

Se efectúa una liberación amplia del muñón pancreático de la vena esplénica. Esta maniobra de liberación del muñón pancreático permite tener una mejor exposición para realizar la anastomosis. $\mathrm{La}$ anastomosis se inicia con un primer plano con puntos transfixiantes al parénquima pancreático (aproximadamente a 1,5 a $2 \mathrm{~cm}$ del límite de sección), este plano se efectúa entre el páncreas y la serosa del yeyuno en U con prolene 3-0. Nuestra modificación consiste en adicionar pledgets ${ }^{\circledR}$ (politetrafluoro-etileno) en los puntos iniciales en el páncreas con la idea de disminuir la posibilidad de desgarro del tejido pancreático. Al finalizar este primer plano no se anuda, ni se cortan las agujas de las suturas. Posteriormente se realiza la apertura en el asa de yeyuno con la punta de aguja del electrobisturí. Se pasan los puntos de la pared posterior de la anastomosis ducto-mucosa con sutura separada de prolene 6-0 o PDS 6-0, sin anudarlos. Una vez que se han dejado tomados los puntos de la pared posterior se procede a aproximar el asa de yeyuno al páncreas y a anudar las suturas en $\mathrm{U}$ de prolene 3-0 del primer plano, sin cortar las agujas de las suturas. Esto aproxima los tejidos y hace que los nudos de la pared posterior del plano ductomucoso se realicen sin tensión. Esto es de especial utilidad en los páncreas blandos (Figuras 1 y 2).

Una vez terminada la pared posterior del plano ducto-mucoso se instala un tutor transanastomótico 4 ó 5 French, que se deja libre en el asa de yeyuno, sin exteriorizarlo. Posteriormente, se completa la pared anterior de la misma manera que la pared posterior. La anastomosis se termina con un plano que va desde el páncreas, desde donde se anudó el primer plano de prolene 3-0 hasta la serosa del yeyuno, con la idea de producir la invaginación del yeyuno al páncreas (Figuras 2, 3 y 4).

Se completa la reconstrucción realizando la hepatoyeyuno-anastomosis término-lateral y posteriormente la gastro-yeyuno-anastomosis término-lateral, dejando un drenaje tipo Jackson-Pratt en relación a la anastomosis pancreática y biliar.

No se deja sonda nasoyeyunal, ni yeyunostomia de alimentación de rutina.

\section{Variables de estudio}

Se registraron variables biodemográficas, quirúrgicas y de evolución clínica.

Se registró la presencia de fístula pancreática y el 


\section{ARTÍ́CULO ORIGINAL}

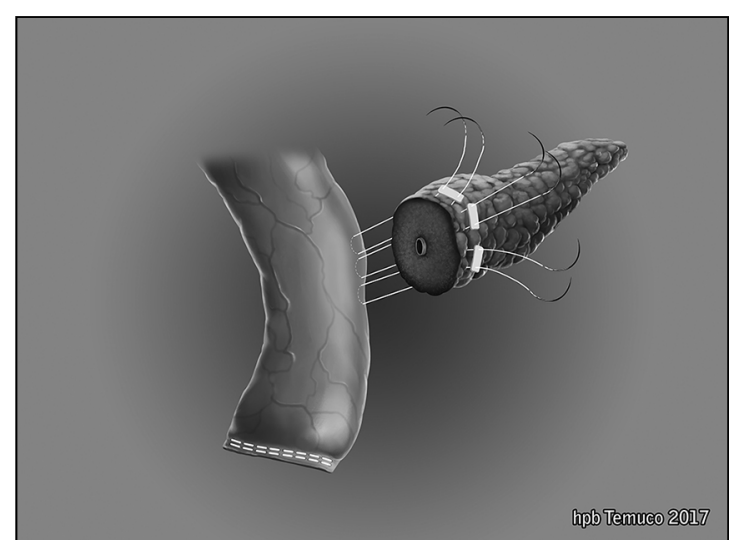

Figura 1. Primer plano con puntos transfixiantes al parénquima pancreático (aproximadamente 1,5 a $2 \mathrm{~cm}$ del límite de sección). Este plano se efectúa entre el páncreas y la serosa del yeyuno en $U$ con prolene 3-0. Se usa pledgets ${ }^{\circledR}$ (poli-tetrafluoro-etileno) en el lado del páncreas, y no se anuda ni se cortan las agujas.

riesgo de presentar fístula pancreática según score de Callery y cols ${ }^{14}$.

Se clasificó el estadio del cáncer según $\mathrm{TNM}^{15}$.

\section{Definiciones}

Fístula pancreática posoperatoria (FPPO): Se definió y clasificó fístula pancreática de acuerdo a la clasificación del International Study Group of Pancreatic Fistulae (ISGPF) ${ }^{16}$, como cualquier drenaje medible de una operación (o drenaje percutáneo colocado posteriormente) a partir del tercer día posoperatorio, con una amilasa en el contenido de drenaje mayor a tres veces el valor normal de amilasa sérica (> $300 \mathrm{UI} / 1)$. Todos los pacientes que tengan un valor bajo este límite, fueron considerados sin fístula. Se divide en 3 grados de severidad según criterios clínicos:

Grado A: Fístula transitoria, asintomática, sólo con elevación de amilasa en el drenaje. No requiere tratamiento específico, ni reoperación. Drenaje se retira en 3 semanas o menos. Se considera sin relevancia clínica.

Grado B: Fístula sintomática, clínicamente requiere evaluación y tratamiento específico. Puede requerir reoperación. El drenaje permanece más de 3 semanas.

Grado $C$ : Fístula grave, puede comprometer la vida. Frecuentemente requiere reoperación y tratamiento de soporte.

Los grados $\mathrm{B}$ y $\mathrm{C}$ se consideran actualmente como clínicamente relevantes ${ }^{17}$, y éstas son las que consideramos al evaluar la morbilidad posoperatoria.

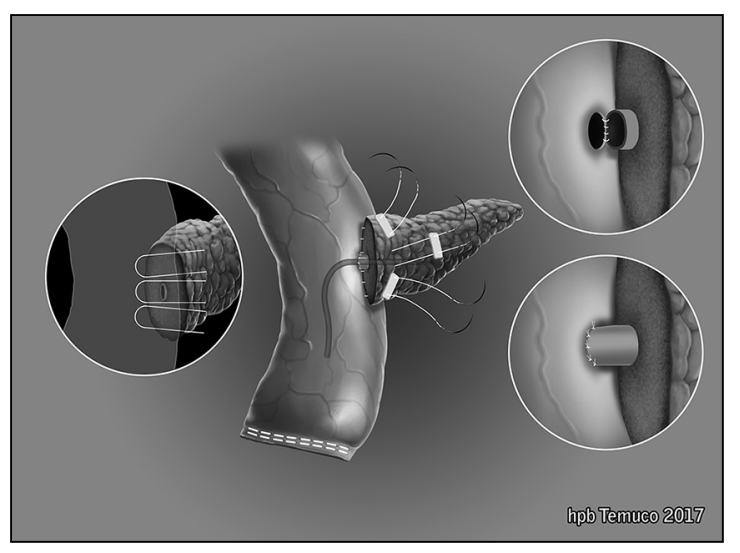

Figura 2. Segundo plano ducto-mucosa (pared posterior) con puntos individuales de prolene 6-0.

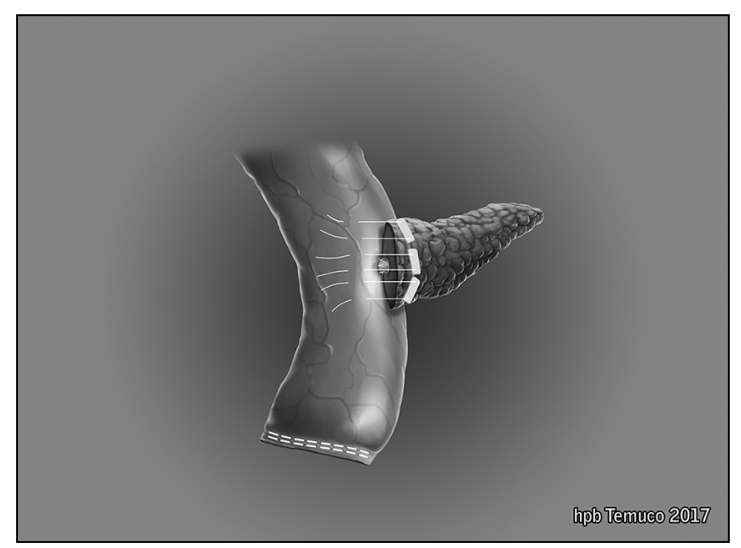

Figura 3. Plano proveniente de la serosa del páncreas a la pared de yeyuno con la idea de producir invaginación del páncreas al yeyuno.

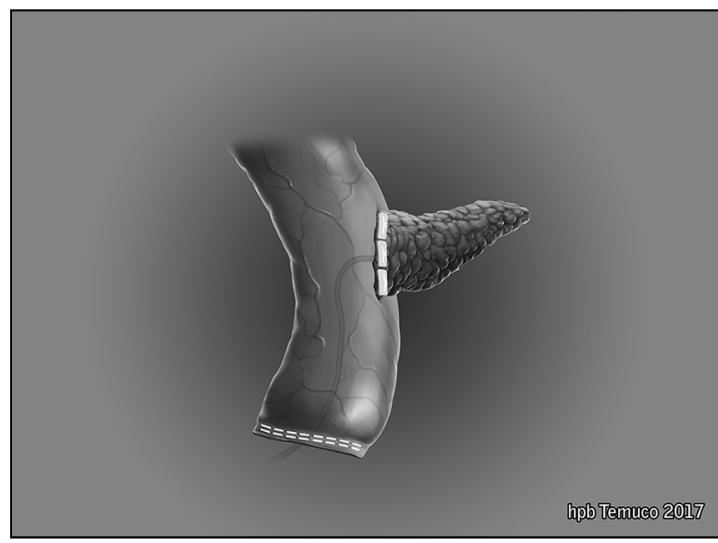

Figura 4. Visión final de la anastomosis. Se visualiza el tutor en el asa de yeyuno.
Score de riesgo de fistula: Se calcula según textura del páncreas, patología, diámetro del conducto pancreático y pérdidas hemáticas intraoperatorias, según score de Callery y cols ${ }^{14}$.

Morbilidad: Se definió morbilidad peri-operatoria según la clasificación de Clavien-Dindo ${ }^{18}$.

Mortalidad: Se midió mortalidad como variable dicotómica. 


\section{ARTÍCULO ORIGINAL}

Herramientas estadísticas: Se utilizó estadística descriptiva con medidas de tendencia central y dispersión. Se diseñó la base de datos en Excel $2017^{\circledR}$ y el análisis se realizó en el programa Stata 12.0 .

\section{Resultados}

La serie está compuesta por 12 pacientes, 9 (75\%) de género femenino y $3(25 \%)$ de género masculino.

La edad promedio de la serie total fue de $59 \pm 8,5$ años. $59 \pm 9,3$ y $57 \pm 6,6$ años, en género femenino y masculino, respectivamente.

Con respecto a motivo inicial de consulta, 9 pacientes $(75 \%)$ presentaron ictericia obstructiva al momento de consultar, 2 pacientes $(16,6 \%)$ debutaron como pancreatitis aguda y 1 paciente $(8,33 \%)$ como dolor abdominal posderivación biliodigestiva.

En cuanto a ASA score, 10 pacientes $(83,3 \%)$ presentaron ASA II al momento de la cirugía y 2 pacientes $(16,6 \%)$ ASA I.

El promedio de albúmina preoperatoria de la serie fue de $3,7 \pm 0,7 \mathrm{~g} / \mathrm{dL} .3$ pacientes $(25 \%)$ presentaron albúmina bajo 3,5 , con un límite inferior de 2,0 .

Tabla 1. Características generales de la serie

\begin{tabular}{|ll|}
\hline Variable & \\
\hline Edad (promedio \pm DE, años) & $59 \pm 8,5$ \\
\hline Género femenino (\%) & 75 \\
\hline Ictericia (\%) & 75 \\
\hline ASA II (\%) & 83,3 \\
\hline Albúmina (promedio \pm DE, g/dL) & $3,7 \pm 0,7$ \\
Bilirrubina Total (promedio \pm DE, mg/dL) & $11,4 \pm 9,3$ \\
Bilirrubina Directa (promedio \pm DE, mg/dL) & $8,8 \pm 7,3$ \\
GOT (promedio \pm DE, U/L) & $80,4 \pm 7,3$ \\
GPT (promedio \pm DE, U/L) & $81,4 \pm 65,2$ \\
Tiempo quirúrgico (promedio \pm DE, min) & $329 \pm 55,9$ \\
Enfermedad maligna en biopsia preoperatoria (\%) & 66,6 \\
Ganglios resecados (promedio \pm DE) & $8 \pm 5$ \\
\hline Fístula pancreática clínicamente relevante (\%) & 33,3 \\
Hospitalización (promedio \pm DE, días) & $15,5 \pm 8,6$ \\
\hline Morbilidad (\%) & 25 \\
Mortalidad (\%) & 0 \\
\hline
\end{tabular}

El promedio de bilirrubina total preoperatoria de la serie fue de $11,4 \pm 9,3 \mathrm{mg} / \mathrm{dL}$, y promedio de bilirrubina directa fue de $8,8 \pm 7,3 \mathrm{mg} / \mathrm{dL}$. El promedio de GOT de ingreso fue de 80,4 $\pm 74,3 \mathrm{U} / \mathrm{L}, \mathrm{y}$ el promedio de GPT de ingreso fue de 81,4 $\pm 65,2$ $\mathrm{U} / \mathrm{L}$. El promedio de fosfatasas alcalinas de ingreso fue de $472,6 \pm 347,4 \mathrm{U} / \mathrm{L}$.

Del total de pacientes, 10 pacientes contaban con ecografía abdominal con dilatación de vía biliar, 9 pacientes con tomografía computada de abdomen $\mathrm{y}$ pelvis, 5 pacientes con colangioresonancia, y 4 pacientes con resonancia nuclear magnética de abdomen.

El tiempo quirúrgico total (desde la inducción anestésica hasta el término de la anestesia) promedio de la serie fue de $329 \pm 55,9 \mathrm{~min}$.

Ocho pacientes $(66,6 \%)$ tenían biopsia preoperatoria compatible o sugerente de enfermedad maligna (Tabla 1); 6 pacientes mediante colangiografía endoscópica retrógrada y 2 pacientes mediante endoscopia digestiva alta.

Todos los pacientes tenían confirmación de lesión neoplásica en la biopsia operatoria; 10 de ellos $(83,3 \%)$ correspondían a cáncer de ampolla de Váter, y 2 pacientes $(16,6 \%)$ a tumor de colédoco distal.

El $100 \%$ de los pacientes presentaron como tipo histológico adenocarcinoma.

El promedio de ganglios resecados fue de $8 \pm 5$.

En cuanto a la estadificación, 2 pacientes $(16,6 \%)$ fueron clasificados como T1, 4 pacientes $(33,3 \%)$ como T2, 3 pacientes $(25 \%)$ como T3 y 3 pacientes $(25 \%)$ como T4. 4 pacientes $(33,3 \%)$ tuvieron ganglios positivos (N1).

Del total de la serie, 4 pacientes $(33,3 \%)$ presentaron fístula pancreática grado $\mathrm{A}$, sin relevancia clínica, por lo tanto, no incluida dentro de morbilidad importante. No hubo presencia de fístula grado $\mathrm{B} / \mathrm{C}$ en nuestra serie.

Tabla 2. Morbilidad de Clavien-Dindo

\begin{tabular}{|llc|}
\hline Grado & n & \% \\
\hline 0 & 9 & 75 \\
I & 0 & 0 \\
\hline II & 2 & 16,6 \\
\hline IIIa & 1 & 8,33 \\
\hline IIIb & 0 & 0 \\
\hline IV & 0 & 0 \\
\hline V & 0 & 0 \\
\hline
\end{tabular}


ARTíCULO ORIGINAL

Restando este aspecto, 9 pacientes no presentaron otro tipo morbilidad. Dentro de la morbilidad quirúrgica: 2 pacientes $(16,6 \%)$ presentaron infección de herida operatoria superficial y 1 paciente $(8,3 \%)$ presentó evisceración cubierta y hemorragia digestiva alta secundaria a pseudoaneurisma de arteria gastroduodenal que fue tratada con embolización. La gravedad de la morbilidad evaluada según clasificación de Clavien-Dindo se puede observar en la Tabla 2. Nueve pacientes (75\%) no presentaron morbilidad.

En la Tabla 3 se describe el score de riesgo de fístula pancreática según sus variables. Al clasificar la serie según esto: 4 pacientes $(33,3 \%)$ presentaron bajo riesgo, 7 pacientes $(58,3 \%)$ riesgo intermedio, y 1 paciente $(8,3 \%)$ alto riesgo de presentar FPPO.

Según score de riesgo de FPPO, 3 pacientes (25\%) del grupo de riesgo intermedio desarrolló fístula pancreática y 1 paciente $(8,3 \%)$ del grupo de bajo riesgo. El único paciente con alto riesgo no presentó fístula pancreática (Tabla 4).

El promedio de días de hospitalización fue de 15,5 $\pm 8,6$ días (Tabla 1).

No hubo mortalidad posoperatoria en nuestra serie. No hubo readmisiones luego del alta.

El seguimiento promedio de la serie fue de 9,7 $\pm 9,7$ meses. Al momento del reporte están todos los pacientes vivos, sin evidencia de disfunción exocrina del páncreas, ni evidencia de obstrucción de la anastomosis.

\section{Discusión}

La mayor tasa de morbilidad y mortalidad en PD está relacionada con las complicaciones de la reconstrucción pancreática ${ }^{4-6}$, y existen múltiples opciones para realizarla.

Nuestro equipo publicó un primer reporte en el año 2012 con el uso de PGADM, con morbilidad global del 35\% y mortalidad del 5\%. Sin embargo, aunque esta técnica es segura y reproducible, nos enfrentamos a 2 potenciales problemas teóricos: la fístula pancreática de alto débito y la posibilidad de dehiscencia al efectuar control endoscópico de hemorragia digestiva alta. La evidencia científica disponible es contradictoria al evaluar estos aspectos ${ }^{19,20}$

Nuestros pacientes tienen estadías preoperatorias prolongadas, con cuadros infecciosos previos que aumentan el riesgo de lesiones de estrés en la mucosa gástrica y/o duodenal.

Es por eso que, en la búsqueda de la mejor técnica de reconstrucción, encontramos la técnica de
Tabla 3. Score de fístula pancreática ${ }^{14}$, desglosado según variables independientes

\begin{tabular}{|llc|}
\hline \multicolumn{2}{|l|}{ Variables score de fístula pancreática } & n de pacientes \\
Páncreas & Duro & 4 \\
& Blando & 8 \\
Histopatología & Adenocarcinoma pancreático o pancreatitis & 0 \\
& Ampular, duodenal, cístico, célula de islote & 12 \\
Diámetro del conducto & $\geq 5$ & 6 \\
de Wirsung & 4 & 4 \\
& 3 & 1 \\
& 2 & 0 \\
Pérdidas hemáticas & $\leq 1$ & 1 \\
intraoperatorias, $\mathrm{mL}$ & $\leq 400$ & 12 \\
& $401-700$ & 0 \\
& $701-1.000$ & 0 \\
\hline
\end{tabular}

Tabla 4. Pacientes con score de riesgo de fístula pancreática y presencia de fístula según este

\begin{tabular}{|lcc|}
\hline Grupos de riesgo & $\begin{array}{c}\text { Riesgo de fístula }(\%) \\
(\mathbf{n}=\mathbf{1 2})\end{array}$ & Fístula pancreática \\
\hline Despreciable & 0 & 0 \\
Bajo & $4(33,3)$ & 1 \\
Intermedio & $7(58,3)$ & 3 \\
Alto & $1(8,3)$ & 0 \\
\hline
\end{tabular}

Blumgart que había mostrado ser segura y reproducible en algunos reportes ${ }^{21,22}$. Aunque el número de pacientes es bajo, consideramos que puede ser una técnica importante a tener en cuenta en centros que realizan $\mathrm{PD}$ en el país.

El más reciente ensayo clínico aleatorizado multicéntrico respecto a la reconstrucción pancreática post-PD (estudio RECOPANC) mostró una tasa global de fístula pancreática $\mathrm{B}$ o $\mathrm{C}$ de $21 \%$ y una mortalidad hospitalaria de $6 \%$. No hubo diferencia respecto a la tasa de fístulas pancreáticas $\mathrm{B}$ o $\mathrm{C}$ al comparar la pancreatogastrostomía con la pancreatoyeyunostomía; sin embargo, hubo más episodios de hemorragia posoperatoria en el grupo de pancreatogastrostomía ${ }^{23}$.

En las sesiones de simulación, previo a poner en práctica la técnica, surgió la idea de colocar pledgets en el lado pancreático para disminuir la posibilidad de desgarro del tejido pancreático al anudar. La técnica de Blumgart combina las ventajas de la invaginación del páncreas en el yeyuno y la anastomosis ducto-mucosa.

Nuestro equipo utiliza siempre magnificación 
para realizar la reconstrucción en la PD y la realización de los nudos de las suturas se hacen con técnica que permite disminuir la tensión y la posibilidad de desagarro del tejido pancreático ${ }^{24}$. A esto también contribuye la modificación de usar los pledgets.

La morbilidad de nuestra serie es aceptable $(25 \%)$, siendo la mayoría grado II en la escala Clavien-Dindo. Las cifras de morbilidad global asociadas a PD en algunos reportes puede alcanzar el $50 \%$ 4,5,6,11,25.

La cifra de fístulas pancreáticas de esta serie fue de $33,3 \%$, todas las fístulas fueron grado A, considerada sin relevancia clínica y muchos centros no incluyen este tipo de fístulas dentro de la morbilidad importante en PD. Si bien esta cifra es más alta que la reportada por nuestro grupo en un reporte de reconstrucción utilizando $\mathrm{PGADM}^{11}$, en la presente serie con reconstrucción con técnica de Blumgart no hay mortalidad. Esto ha sido reportado por varios estudios que muestran una menor tendencia a desarrollar FP con las reconstrucciones pancreatogástricas.

Una de las ventajas de la reconstrucción con técnica de Blumgart es que permite alimentar al paciente precozmente y disminuir el tiempo de estadía hospitalaria. En este aspecto no fue posible efectuar comparación directa, ya que en nuestro reporte previo no se describió este aspecto. Sin embargo, nuestro promedio de estancia hospitalaria $(15,5 \pm$ 8,6 días) es similar al promedio de estancia hospitalaria reportado por Chan (14 días) en el reporte del Instituto Nacional de Ciencia y Nutrición Salvador Zubirán de México $^{26}$.

La mayoría de las fístulas pancreáticas (3/4) se presentaron en pacientes con escalas de riesgo intermedio de fístula. La consistencia del páncreas y el diámetro del Wirsung son dos de los más importantes factores locales para el desarrollo de FP. El desarrollo de una fístula pancreática en el grupo de riesgo bajo y el no desarrollo de fístulas en el grupo de alto riesgo solo muestra que los sistemas de predicción no son perfectos y que el desarrollo de FP depende de múltiples factores.

Nuestro grupo no utiliza terapia farmacológica para prevención de fístula con pasireotide por su costo, aunque hay un ensayo clínico aleatorio que demostró su utilidad en reducción de fístula pancreática pospancreatectomía ${ }^{27}$.

El hallazgo de 10 pacientes con tumor de ampolla de Vater y 2 con colangiocarcinoma distal es debido al azar, se asocia posiblemente al número de pacientes, y no corresponde a un criterio de selección.

Las limitaciones de nuestro trabajo son el diseño de serie de casos y el tamaño de la muestra.

Teniendo en cuenta estas limitaciones, nuestro trabajo muestra que la técnica de Blumgart modificada es reproducible, con una morbilidad aceptable y puede ser una alternativa en la reconstrucción pancreática post-PD. A pesar de que la tasa de FPPO fue más alta en el reporte previo de nuestro grupo, utilizando PGADM, en el presente estudio no hubo desarrollo de FPPO clínicamente relevante. Estos resultados iniciales tienen que ser demostrados al aumentar el número de casos.

\section{Responsabilidades éticas}

Protección de personas y animales. Los autores declaran que para esta investigación no se han realizado experimentos en seres humanos ni en animales.

Confidencialidad de los datos. Los autores declaran que han seguido los protocolos de su centro de trabajo sobre la publicación de datos de pacientes.

Derecho a la privacidad y consentimiento informado. Los autores declaran que en este artículo no aparecen datos de pacientes.

\section{Financiación}

Los autores declaran no haber recibido ninguna financiación para la realización de este trabajo.

\section{Conflicto de intereses}

Los autores declaran no tener ningún conflicto de intereses.

\section{Bibliografía}

1. Van Heek NT, Kuhlmann KF, Scholten RJ, de Castro SM, Busch OR, van Gulik TM, et al. Hospital volumen and mortality after pancreatic resection: a systematic review and an evaluation of intervention in the
Netherlands. Ann Surg. 2005;242:781-8.

2. Aumont O, Dupré A, Abjean A, Pereira B, Veziant J, Le Roy B, et al. Does Intraoperative closed-suction drainage influence the rate of pancreatic fistula after pancreatoduodenectomy? BMC Surgery 2017;17:58.
3. Perinel J, Mariette C, Dousset B, Sielezneff I, Gainant A, Mabrut JY, et al. Early enteral versus total parenteral nutrition in patients undergoing pancreaticoduodenectomy: A randomized multicenter controlled trial (Nutri-DPC). Ann Surg. 2016;264:731-7. 
4. Norero E, Viñuela E, Báez S, Martínez C, Reyes J, Kusanovic R, et al. Resultados actuales de la pancreatoduodenectomía para el tratamiento de los tumores periampulares y análisis de factores pronósticos de sobrevida. Rev Med Chile 2011;139:1015-24.

5. Waugh E. Pancreatogastroanastomosis en pancreatoduodenectomía. Una alternativa segura de anastomosis al muñón pancreático. Rev Chil Cir. 2002;54:59-64.

6. Burmeister R, Benavides C, García C, Perales C. Duodenopancreatectomía con conservación de píloro. Rev Chil Cir. 2001;53:164-9

7. Gouma DJ, van Geenen RC, van Gulik TM, de Haan RJ, de Wit LT, Busch OR, et al. Rates of complications and death after pancreaticoduodenectomy: risk factors and the impact of hospital volume. Ann Surg. 2000; 232:786-95.

8. Van Geen RC, Gouma DJ. Impact of hospital volume on in-hospital mortality in pancreatic surgery. Surg Technol Int. 2002;10:61-5.

9. Schmidt CM, Turrini O, Parikh P, House MG, Zyromski NJ, Nakeeb A, et al. Effect of hospital volumen, surgeon experience, and surgeon volumen on patient outcomes after pancreaticoduodenectomy: a single-institution experience. Arch Surg. 2010;145:634-40.

10. Khithani A, Christian D, Lowe K, Saad AJ, Linder JD, Tarnasky Pet al. Feasibility of pancreatoduodenectomy in a nonuniversity tertiary care center: what are the key elements of success? Am Surg. 2011;77:545-51.

11. Losada H, Muñoz C, Contreras J, Burgos L, Silva J. Técnica y resultados con reconstrucción pancreato-gástrica ductomucosa en pancreatoduodenectomía. Rev Chil Cir. 2012;63:257-63.

12. Yang SH, Dou KF, Sharma N, Song WJ. The methods of reconstruction of pancreatic digestive continuity after pancreatoduodenectomy: a meta-analysis of randomized controlled trials. World J Surg. 2011;25:2290-7.

13. Crippa S, Cirocchi R, Randolph J, Partelli S, Belfiori G, Piccioli A, et al. Pancreaticojejunostomy is comparable to pancreaticogastrostomy after pancreaticoduodenectomy: an updated meta-analysis of randomized controlled trials. Langenbecks Arch Surg. 2016;401:427-37.

14. Callery MP, Pratt WB, Kent TS, Chaikof EL, Vollmer CM Jr. A prospectively validated clinical risk score accurately predicts pancreatic fistula after pancreaticoduodenectomy. J Am Coll Surg. 2013;216:1-14.

15. International Union Against Cancer, Sobin LH, Gospodarowicz MK, Wittekind C. TNM classification of malignant tumours. 7th edition, 2009. Pag 1-13.

16. Pratt WB, Maithel SK, Vanounou T, Huang ZS, Callery MP, Vollmer CM Jr. Clinical and economic validation of the International Study Group of Pancreatic Fistula (ISGPF) classification scheme. Ann Surg. 2007;245:443-51.

17. McMillan MT, Soi S, Asbun HJ, Ball CG, Bassi C, Beane JD, et al. Riskadjusted Outcomes of Clinically Relevant Pancreatic Fistula Following Pancreatoduodenectomy: A Model for Performance Evaluation. Ann Surg. 2016;264:344-52.

18. Clavien PA, Barkun J, de Oliveira ML, Vauthey JN, Dindo D, Schulick RD, et al. The Clavien-Dindo classification of surgical complications: five-year experience. Ann Surg. 2009;250:187-96

19. Clerveus M, Morandeira-Rivas A, Picazo-Yeste J, Moreno-Sanz C. Pancreaticogastrostomy versus pancreaticojejunostomy after pancreaticoduodenectomy: a systematic review and meta-analysis of randomized controlled trials. J Gastrointest Surg.
2014;18:1693-704

20. Shrikhande SV, Sivasanker M, Vollmer $\mathrm{CM}$, Friess H, Besselink MG, Fingerhut A, et al. Pancreatic anastomosis after pancreatoduodenectomy: A position statement by the International Study Group of Pancreatic Surgery (ISGPS). Surgery 2017;161:1221-34.

21. Wang SE, Chen SC, Shyr BU, Shyr YM. Comparison of Modified Blumgart pancreaticojejunostomy and pancreaticogastrostomy after pancreaticoduodenectomy. HPB (Oxford) 2016;18:229-35.

22. Kim DJ, Paik KY, Kim W, Kim EK. The effect of modified pancreaticojejunostomy for reducing the pancreatic fistula after pancreaticoduodenectomy. Hepatogastroenterology 2014;61:1421-5.

23. Keck T, Wellner UF, Bahra M, Klein F, Sick O, Niedergethmann $\mathrm{M}$, et al. Pancreatogastrostomy Versys Pancreatojejunostomy for RECOnstruction After PANCreatoduodenectomy (RECOPANC, DRKS 00000767): Perioperative and Long-term Results of a Multicenter Randomized Controlled Trial. Ann Surg. 2016;263:440-9.

24. Neychev VK, Saldinger PF. Minimizing Shear and Compressive Stress During Pancreaticojejunostomy. Rationale of a New Technical Modification. JAMA Surg. 2014;149:203-7.

25. Mohammed S, Fisher W. Quality Metrics in Pancreatic Surgery. Surgical Clinics 2013;93:693-709.

26. Chan C, Franssen B, Uscanga L, Robles G, Campuzano M. Pancreaticoduodenectomía: resultados en un centro de alto volumen. Rev Gastroenterol Mex. 2006;71:252-6.

27. Allen PJ, Gönen M, Brennan MF, Bucknor AA, Robinson LM, Pappas MM, et al. Pasireotide for Postoperative Pancreatic Fistula. N Engl J Med. 2014;370:2014-22. 